VOL. $50(1995) \quad[373-382]$

\title{
FREE LIE ALGEBRA AND LAMBDA-RING STRUCTURE
}

\section{MaRIa RONCO}

Let $R$ be a graded $\lambda$-ring. We extend a well-known formula in the universal ring of Witt vectors by replacing the power operations by the Adams operations. Our method provides us an easy way to compute the inverse image by the symmetric power operators of certain elements of $R$. As a corollary we get identities, found by Klyachko and Hanlon, in the rings $1+\mathbb{Z}[[t]]^{+}$and $1+\widehat{R}[[t]]^{+}$, where $R$ is the representation ring of the symmetric groups.

In their work [1] Dress and Siebeneicher proved the Cyclotomic Identity (see [7]), which takes place in the ring $1+\mathbb{Z}[[t]]^{+}$, by using the Burnside ring and the universal ring of Witt vectors. The main result of this paper is to generalise this identity by proving a similar identity, which takes place in $1+\widehat{R}[[t]]^{+}$, where $R$ is a $\lambda$-ring. In this new setting the $n$-th power operation is replaced by the Adams operation $\Psi^{n}$.

This new formula permits us to give a quick proof of two results, one by Hanlon [3] and one by Klyachko [4], about the representations of the symmetric group associated to the free Lie algebra. Here the $\lambda$-ring $R$ is the representation ring of the symmetric groups $\bigoplus_{n \geqslant 0} R\left(\mathbb{S}_{n}\right)$.

In Section 1 we construct a diagram associated to any graded $\lambda$-ring $R$, that leads us to find the inverse image by $\mathbf{S}=\sum_{i \geqslant 1} S^{i}$ ( $S^{i}$ denotes the symmetric power operator) of certain elements of $R$. We use elementary concepts and results of $\lambda$-ring theory and some ideas of a nice construction of Dress-Siebeneicher (see [1]). In Section 2 we apply the results of Section 1 to the representation ring $R=\bigoplus_{n \geqslant 0} R\left(\mathbb{S}_{n}\right)$.

\section{Primitive elements in a $\lambda$-Ring}

We recall some classical notation and results. For complete definitions and proofs we refer to Knutson (see [5]).

Let $R$ be a torsion free graded $\lambda$-ring, that is $R=\bigoplus_{n \geqslant 0} R_{n}$ is such that its $\lambda$ operations satisfy $R_{i n} \supset \lambda^{i}\left(R_{n}\right)$, for $i, n \geqslant 0$. The ring $\widehat{R}:=\prod_{n \geqslant 0} R_{n}$ has a natural $\lambda$-ring structure, inherited from the one of $R$.

Received 9th December, 1994

Copyright Clearance Centre, Inc. Serial-fee code: 0004-9729/95 \$A2.00+0.00. 
Recall that, in any $\lambda$-ring, the Adams operations $\Psi^{n}$ and $\lambda$-operations $\lambda^{n}$ are related by the formula:

$$
\left(\frac{d}{d t}\right) \log \left(\sum_{i \geqslant 0} \lambda^{i}(a) t^{i}\right)=\sum_{n \geqslant 0}(-1)^{n} \Psi^{n+1}(a) t^{n}, \text { for } a \in \widehat{R}
$$

where $t$ is an indeterminate.

In order to simplify our formulae we work with the "symmetric powers" operations associated to the $\lambda^{i}$ 's, namely the operations $S^{i}$ defined by:

$$
\left[\sum_{i \geqslant 0} \lambda^{i}(a)(-t)^{i}\right]^{-1}=\sum_{n \geqslant 0} S^{n}(a) t^{n}, \text { for } a \in \widehat{R} .
$$

So, (1) and (1') imply:

$$
\left(\frac{d}{d t}\right) \log \left(\sum_{i \geqslant 0} S^{i}(a) t^{i}\right)=\sum_{n \geqslant 0} \Psi^{n+1}(a) t^{n}, \text { for } a \in \widehat{R} .
$$

Denote by $1+\widehat{R}[[t]]^{+}$the multiplicative group of formal power series with coefficients in $\widehat{R}$ and with constant term 1. Equation (2) implies the existence of a commutative diagram:

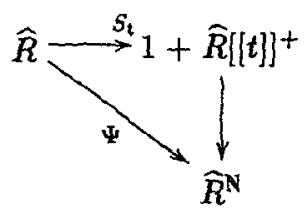

where $S_{t}(a)=1+\sum_{i \geqslant 1} S^{i}(a) t^{i}$, and $\Psi(a)=\left\{\Psi^{n}(a)\right\}_{n \in \mathbb{N}}$, for $a \in \widehat{R}$.

Dress and Siebeneicher show in [1] that well-known product decompositions of formal power series arise from classical isomorphisms between the Burnside ring of the infinite cyclic group on one hand, the Grothendieck ring of formal power series with constant term 1 and the universal ring of Witt vectors of $\mathbb{Z}$, on the other hand. Our goal is to find analogous decompositions in the group of formal power series $1+\widehat{R}[[t]]^{+}$, so we extend formula (2) by mimicking, in a certain way, the Dress-Siebeneicher construction. The idea is to replace the powers in the classical construction of the universal ring of Witt vectors by the Adams operations of the $\lambda$-ring, to get the following: 
THEOREM 1. Let $R$ be a torsion free graded $\lambda$-ring and denote by $\widehat{R}$ the $\lambda$-ring $\prod_{n \geqslant 0} R_{n}$. The following is a commutative diagram of abelian groups:

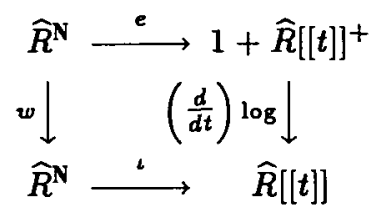

where:

$\widehat{R}^{\mathrm{N}}$ is the group of all maps $\mathbb{N} \rightarrow \widehat{R}$ provided with addition defined componentwise, $\widehat{R}[t]]$ is the additive group of formal power series on $\widehat{R}$,

$$
\begin{aligned}
& e\left(q_{1}, q_{2}, \ldots, q_{n}, \ldots\right):=\prod_{d \geqslant 1}\left(\sum_{i \geqslant 0} t^{d i} S^{i}\left(q_{d}\right)\right), \\
& \iota\left(a_{1}, a_{2}, \ldots, a_{n}, \ldots\right)=\sum_{j \geqslant 0} a_{j+1} t^{j}, \\
& \frac{d}{d t} \log \left(1+\sum_{i \geqslant l} b_{i} t^{i}\right)=\left(\sum_{i \geqslant 1} i b_{i} t^{i-1}\right)\left(1+\sum_{i \geqslant 1} b_{i} t^{i}\right)^{-1} \text { and } \\
& w\left(q_{1}, q_{2}, \ldots, q_{n}, \ldots\right):=\left\{\sum_{d \mid n} d \Psi^{n / d}\left(q_{d}\right)\right\}_{n \in \mathrm{N}} .
\end{aligned}
$$

Proof: The properties of the Adams and $\lambda$-operations:

$$
\begin{aligned}
& S^{i}\left(q+q^{\prime}\right)=\sum_{j+k=i} S^{j}(q)+S^{k}\left(q^{\prime}\right), \text { and } \\
& \Psi^{i}\left(q+q^{\prime}\right)=\Psi^{i}(q)+\Psi^{i}(q ;), \text { for } i \geqslant 1 \text { and } q, q^{\prime} \in \widehat{R},
\end{aligned}
$$

imply that all the applications of the diagram are group homomorphisms.

Let $\mathbf{q}=\left(q_{1}, q_{2}, \ldots, q_{n}, \ldots\right)$ be an element of $\widehat{R}^{\mathrm{N}}$. On one hand one has:

$$
\left\llcorner\circ w(\mathbf{q})=\sum_{n \geqslant 1}\left(\sum_{d \mid n} d \Psi^{n / d}\left(q_{d}\right)\right) t^{n-1}\right.
$$


and on the other hand:

$$
\begin{aligned}
\left(\frac{d}{d t}\right) \log \circ e(\mathbf{q}) & =\left(\frac{d}{d t}\right) \log \left(\prod_{l \geqslant 1}\left(\sum_{j \geqslant 0} t^{j l} S^{j}\left(q_{l}\right)\right)\right) \\
& =\sum_{l \geqslant 1}\left(\frac{d}{d t}\right) \log \left(\sum_{j \geqslant 0}\left(t^{l}\right)^{j} S^{j}\left(q_{l}\right)\right) \quad(\text { by }(2)) \\
& =\sum_{l \geqslant 1}\left(\sum_{n \geqslant 0} \Psi^{n+1}\left(q_{l}\right)\left(t^{l}\right)^{n}\right) l t^{l-1}=\sum_{l \geqslant 1} l \Psi^{n}\left(q_{l}\right) t^{n l-1} \\
& =\sum_{m \geqslant 1}\left(\sum_{l \mid m} l \Psi^{m / l}\left(q_{l}\right)\right) t^{m-1}
\end{aligned}
$$

So one gets $\frac{d}{d t} \log 0 e=\iota \circ w$.

It is easy to see that $\iota$ and $e$ are isomorphisms, while $w$ and $\frac{d}{d t} \log$ are injective. When $R \supset \mathbb{Q}$ all these morphisms are isomorphisms.

Following Dress and Siebeneicher, the fact that the Adams operations are ring homomorphisms satisfying:

$$
\Psi^{n} \circ \Psi^{m}=\Psi^{n m}, \text { for } n, m \in \mathbb{N},
$$

implies that $\left(a_{1}, a_{2}, \ldots a_{n}, \ldots\right) \in \widehat{R}^{\mathrm{N}}$ belongs to the image of $w$ if and only if $n$ divides $\sum_{d \mid n} \mu(d) \Psi^{d}\left(a_{n / d}\right)$, for $n \geqslant 1$, where $\mu$ is the Moebius function.

The inverse morphism of $w$, say $w^{-1}: \operatorname{Im} w \rightarrow \widehat{R}^{\mathbf{N}}$ is then given by:

$$
w^{-1}\left(a_{1}, a_{2}, \ldots, a_{n}, \ldots\right)=\left\{1 / n \sum_{d \mid n} \mu(d) \Psi^{d}\left(a_{n / d}\right)\right\}_{n \in \mathbb{N}} .
$$

REMARK 2. If we change $e$ by the morphism:

$$
\bar{e}\left(q_{1}, q_{2}, \ldots q_{n}, \ldots\right)=\left(\prod_{d \geqslant 1}\left(\sum_{i \geqslant 0} t^{d i} \lambda^{i}\left(q_{d}\right)\right)\right),
$$

and $w$ by:

$$
\bar{w}\left(q_{1}, q_{2}, \ldots q_{n}, \ldots\right)=\left\{\sum_{d \mid n} d(-1)^{n / d-1} \Psi^{n / d}\left(q_{d}\right)\right\}_{n \in \mathbb{N}}
$$


then the diagram (3) is still commutative.

Let $f$ be an element of $\widehat{R}$, that is $f=\sum_{n \geqslant 0} f_{n}$, with $\operatorname{deg}\left(f_{n}\right)=n$, for $n \geqslant 0$, such that $f_{0}=1$. There exists a natural way to associate to $f$ a power series $\bar{f}(t) \in$ $1+\widehat{R}[[t]]^{+}$by:

$$
\bar{f}(t)=1+\sum_{n \geqslant 1} f_{n} t^{n}
$$

It is easily seen that $e^{-1}(\bar{f}(t))=\left(l_{1}(f), l_{2}(f), \ldots, l_{n}(f), \ldots\right)$ has the property:

"for each $m \in \mathbb{N}$ there exists a finite number of elements $i \in \mathbb{N}$ such that the component of degree $m$ of $l_{i}(f)$ is different from zero". So, we may define the primitive element of $f, l(f) \in \widehat{R}$, as the sum $\sum_{n \geqslant 1} l_{n}(f)$. Clearly $\sum_{n \geqslant 1} S^{n}(l(f))=f$.

The main point of our construction is its relation with well known identities of $1+$ $\mathbb{Z}[[t]]^{+}$. Given a formal power series $a(t) \in 1+\mathbb{Z}[[t]]^{+}$, there exist uniquely determined infinite sequences (see [1]) $\left\{b_{n}\right\}_{n \in \mathbb{N}},\left\{q_{n}\right\}_{n \in \mathbb{N}}$ and $\left\{d_{n}\right\}_{n \in \mathbb{N}}$ in $\mathbb{Z}^{\mathbb{N}}$ such that

$$
a(t)=\prod_{n \geqslant 1}\left(\sum_{i \geqslant 0} t^{n i}\right)^{b_{n}}=\prod_{n \geqslant 1}\left(\sum_{i \geqslant 0} q_{n}^{i} t^{n i}\right)=\exp \left(\sum_{n \geqslant 1} \frac{d_{n}}{n} t^{n}\right) .
$$

The relations between $\left\{b_{n}\right\}_{n \in \mathbb{N}},\left\{q_{n}\right\}_{n \in \mathbb{N}}$ and $\left\{d_{n}\right\}_{n \in \mathbb{N}}$ are given by:

$$
d_{n}=\sum_{l \mid n} l b_{l}=\sum_{l \mid n} l q_{l}^{n / l}, \text { and } b_{n}=1 / n \sum_{l \mid n} \mu(l) d_{n / l}, \text { for } n \geqslant 1 .
$$

Lemma 3. Let $f \in \widehat{R}$ and let $\left\{b_{n}\right\}_{n \in \mathbb{N}},\left\{q_{n}\right\}_{n \in \mathbb{N}}$ and $\left\{d_{n}\right\}_{n \in \mathbb{N}}$ be sequences in $\mathbb{Z}^{\mathbb{N}}$ satisfying (4). Then we have:

$$
\begin{aligned}
& \text { (a) } \prod_{n \geqslant 1}\left(\sum_{i \geqslant 0} q_{n}^{i} S^{i} \circ \Psi^{n}(f)\right)=\exp \left(\sum_{n \geqslant 1} \frac{d_{n}}{n} \Psi^{n}(f)\right) . \\
& \text { (b) } \prod_{n \geqslant 1}\left(1+\sum_{r \geqslant 1} \Psi^{n}\left(f_{r}\right)\right)^{b_{n}}=\exp \left(\sum_{n \geqslant 1} \frac{d_{n}}{n} \Psi^{n}(l(f))\right) ;
\end{aligned}
$$

where $f_{r}$ denotes the component of degree $r$ of $f$, for $r \geqslant 1$.

Proof: (a) By (2) we have:

$$
\sum_{i \geqslant 0} q_{n}^{i} S^{i} \circ \Psi^{n}(f)=\exp \left(\sum_{n \geqslant 1} \frac{q_{n}^{i}}{i} \Psi^{n i}(f)\right), \text { for } n \geqslant 1 .
$$


So,

$$
\begin{aligned}
\prod_{n \geqslant 1}\left(\sum_{i \geqslant 0} q_{n}^{i} S^{i} \circ \Psi^{n}(f)\right) & =\exp \left(\sum_{\substack{n \\
i \geqslant 1}} \frac{q_{n}^{i}}{i} \Psi^{n i}(f)\right) \\
& =\exp \left(\sum_{m \geqslant 1} 1 / m\left(\sum_{l \mid m} l q_{l}^{m / l}\right) \Psi^{m}(f)\right] \\
& =\exp \left(\sum_{m \geqslant 1} \frac{d_{m}}{m} \Psi^{m}(f)\right) .
\end{aligned}
$$

(b) Let $\left\{g_{n}\right\}_{n \in \mathrm{N}}$ be the sequence of elements of $\widehat{R}$ such that $\log \left(1+\sum_{n \geqslant 1} f_{n} t^{n}\right)=$ $\sum_{n \geqslant 1} g_{n} t^{n}$

Then, $l_{r}(f)=1 / r \sum_{d \mid r} \mu(d) \frac{r}{d} \Psi^{d}\left(g_{r / d}\right)=\sum_{d \mid r} \frac{\mu(d)}{d} \Psi^{d}\left(g_{r / d}\right)$.

Applying the function log to our equality, we get:

$$
\sum_{n \geqslant 1} b_{n} \log \left(1+\sum_{r \geqslant 1} \Psi^{n}\left(f_{r}\right)\right)=\sum_{n \geqslant 1} \frac{d_{n}}{n} \Psi^{n}(l(f)) .
$$

But, $\log \left(1+\sum_{r \geqslant 1} \Psi^{n}\left(f_{r}\right)\right)=\Psi^{n}\left(\log \left(1+\sum_{r \geqslant 1} f_{r}\right)\right)=\Psi^{n}\left(\sum_{r \geqslant 1} g_{r}\right)$. Now

$$
\begin{aligned}
\sum_{n \geqslant 1} & \frac{d_{n}}{n} \Psi^{n}(l(f))=\sum_{n \geqslant 1} \frac{d_{n}}{n} \Psi^{n}\left(\sum_{r \geqslant 1} \sum_{d \mid r} \frac{\mu(d)}{d} \Psi^{d}\left(g_{r / d}\right)\right) \\
= & \sum_{\substack{d \\
r \geqslant 1 \\
d \mid r}} \mu(d) \frac{d_{n}}{n d} \Psi^{n d}\left(g_{r / d}\right) \\
= & \left.\sum_{\substack{d \\
r \geqslant 1 \\
r \geqslant 1}} \mu(d) \frac{d_{n}}{n d} \Psi^{n d}\left(g_{k}\right)=\text { (making } n d=m\right), \\
= & \sum_{\substack{k \geqslant 1 \\
d \mid m}} \mu(d) \frac{d_{m / d}}{m} \Psi^{m}\left(g_{k}\right) \\
= & \sum_{m \geqslant 1} 1 / m\left(\sum_{d \mid m} \mu(d) d_{m / d}\right) \Psi^{m}\left(\sum_{k \geqslant 1} g_{k}\right)=\sum_{m \geqslant 1} b_{m} \log \left(1+\sum_{r \geqslant 1} \Psi^{m}\left(f_{r}\right)\right) .
\end{aligned}
$$


An immediate consequence of formula (b) in Lemma 3 is the formula of the following Corollary, which is similar to the Cyclotomic Identity in $1+\mathbb{Z}[[t]]^{+}$(see $[1]$ or $\left.[7]\right)$ :

Corollary 4 . Let $q \in \mathbb{N}$ and let $M(n, q):=1 / n \sum_{l \mid n} \mu(l) q^{n / l}$ for $n \in \mathbb{N}$. (This is the number of primitive necklaces.) Then for $f \in \widehat{R}$ we have:

$$
\sum_{n \geqslant 0} q^{n} S^{n}(l(f))=\prod_{n \geqslant 1}\left(1+\Psi^{n}(f)\right)^{M(n, q)} .
$$

Proof: Apply Lemma 3 for $\left\{q_{n}\right\}_{n \in \mathbb{N}}$ defined by $q_{1}=q$ and $q_{n}=0$ for $n \geqslant 2$.

\section{The $\lambda$-RING OF REPRESENTATIONS OF THE SYMMETRIC GROUP}

We are going to apply the results of Section 1 to the ring $\mathbf{R}=\underset{n \geqslant 0}{\bigoplus_{0}} R\left(\mathbb{S}_{n}\right)$, where $R\left(\mathbb{S}_{n}\right)$ is the Grothendieck group of complex representations of the symmetric group $\mathbb{S}_{n}$ (see [5]), for $n \geqslant 1$, and $R\left(\mathbb{S}_{0}\right)=\mathbb{Z}$. The ring $\mathbf{R}$ acts on itself (see [8]) in such a way that the $n$-th $\lambda$-operation $\lambda^{n}$ corresponds to the action of the alternate representation $s g n_{\mathbb{S}_{n}}$ of $\mathbb{S}_{n}$, and the operator $S^{n}$ is given by the trivial representation $\mathbf{1}_{\mathbb{S}_{n}}$ of $\mathbb{S}_{n}$, for $n \geqslant 1$.

For $n, d \in \mathbb{N}$ such that $n \mid d$, we denote by $s_{n}^{d}$ the element of $R\left(\mathbb{S}_{n d}\right)$ whose character is given by:

$$
\chi_{n}^{d}(\sigma)= \begin{cases}(n !)^{d} d ! & \text { if } \sigma \text { is the product of } d \text { disjoint cycles of length } n \\ 0 & \text { otherwise }\end{cases}
$$

for $\sigma \in \mathbb{S}_{n d}$.

Then the $n$-th Adams operation $\Psi^{n}$ on $\mathbf{R}$ is identified with the action associated to the elements $s_{n} \in R\left(\mathbb{S}_{n}\right)$.

Denote by $\mathrm{Lie}_{n}$ the representation of $\mathbb{S}_{n}$ such that, for any vector space $V$, the vector space $\left.\operatorname{Lie}_{n}(V)=\left(\operatorname{Lie}_{n}\right) \otimes_{q} s_{n}\right] V^{\otimes n}$ is the degree $n$ part of the free Lie algebra over $V$. We want to calculate the element Lie $:=\prod_{n \geqslant 0} \operatorname{Lie}_{n} \in \widehat{\mathbb{R}}$. The universal enveloping algebra of Lie $(V)$ is the tensor algebra $\mathbf{T}(V)$. The representation $\mathbf{T}$ is the "regular representation" element of $\widehat{\mathbb{R}}$, that is $T=\sum_{n \geqslant 0} \operatorname{Reg}_{\mathbb{S}_{n}}$, where $\operatorname{Reg}_{\mathfrak{S}_{n}}$ is the regular representation of $\mathbb{S}_{n}$, for $n \geqslant 1$. The Poincaré-Birkhoff-Witt Theorem states that $\mathbf{T}$ is the symmetric algebra operator $\mathbf{S}=\sum_{i \geqslant 0} S^{i}$ over Lie. So, the following result of Klyachko (see [4]) appears as an immediate consequence of Theorem 1: 
Corollary 5. The free Lie algebra representation in degree $n, \operatorname{Lie}_{n} \in R\left(\mathbb{S}_{n}\right)$, satisfies:

$$
\operatorname{Lie}_{n}=1 / r \sum_{n \mid d} \mu(d) s_{d}^{n / d},
$$

where $\mu$ is the Moebius function and $s_{d}^{n / d}$ is the element of $R\left(\mathbb{S}_{n}\right)$ defined in (5), for $n \geqslant 1$.

Proof: It suffices to remark that $\operatorname{Reg}_{\mathbb{S}_{n}}=s_{1}^{n}$, for $n \in \mathbb{N}$. So applying our results of Section 1 to $f=\mathbf{T}=\sum_{n \geqslant 0} s_{1}^{n}$ gives Klyachko's formula.

We can also deduce Hanlon's equation (see [3]) for the Eulerian characters:

COROLLARY 6 . The elements $S^{n}($ Lie) satisfy the following equality in $\widehat{\mathbf{R}}$ :

$$
\sum_{n \geqslant 1} q^{n} S^{n}(\mathrm{Lie})=\prod_{n \geqslant 1}\left(\sum_{i \geqslant 0} s_{i}^{n}\right)^{M(n, q)} \quad \text { for } q \in \mathbb{N},
$$

where $M(n, q)=1 / n \sum_{l \mid n} \mu(l) q^{n / l}$.

Proof: Since $\Psi^{i}\left(s_{1}^{n}\right)=s_{i}^{n}$, for $i, n \geqslant 0$, Corollary 4 applied to $f=\sum_{n \geqslant 0} s_{1}^{n}$ gives the desired equality.

Note that this equation allows Hanlon to determine the dimension of $S^{i}(\mathrm{Lie})_{n}$ and the multiplicity of certain irreducible representations of the symmetric group $\mathbb{S}_{n}$ in $S^{i}(\text { Lie })_{n}$, for $n, i \geqslant 1$.

In fact, when we speak about $\mathbf{T}(V)$ we have to point out the difference between two cases:

- the "non-graded" tensor algebra, whose coproduct $\Delta$ is cocommutative, that is $\tau \circ \Delta=\Delta$ where $\tau(x \otimes y)=y \otimes x$, for $x, y \in \mathbf{T}(V)$.

- the "graded" tensor algebra; whose coproducts $\Delta$ is graded cocommutative, that is $\tau \circ \Delta=\Delta$ where $\tau(x \otimes y)=(-1)^{|x||y|} y \otimes x$, for $x, y \in \mathbf{T}(V)$ and $|\boldsymbol{x}|$ the degree of $\boldsymbol{x}$.

Up to now our analysis corresponds to the first case. However when we want to study the representations associated to the Eulerian elements defined by GerstenhaberSchack and Loday (see [2], [3] or [6]) we have to look at the second one. The results then follow from a slight modification of Theorem 1 . Let us denote by $\widehat{\text { Lie }}$ the Lie algebra of primitive elements of the graded tensor algebra. The Poincare-Birkhoff-Witt Theorem says that:

$$
\mathbf{T}=\sum_{i \geqslant 0} S^{i}\left(\bigoplus_{n} \widehat{\mathrm{Lie}}_{n}\right) \otimes \sum_{i \geqslant 0} \lambda^{i}\left(\underset{n \text { odd }}{\bigoplus_{\operatorname{Lie}_{n}}}\right)
$$


To compute $\widehat{\mathrm{Lie}}$ we have Proposition 7 , whose proof is almost the same as the proof of Theorem 1.

Proposition 7. Under the same hypothesis as Theorem 1, the following diagram is commutative:

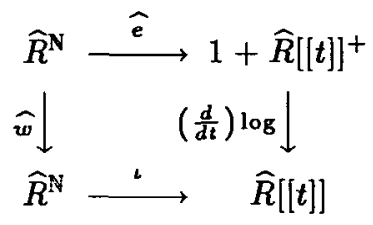

where:

$$
\begin{aligned}
& \iota \text { and } \frac{d}{d t} \log \text { are the same as in Theorem } 1, \\
& \widehat{e}\left(q_{1}, \ldots, q_{n}, \ldots\right)=\left[\prod_{d \text { even }}\left(\sum_{i \geqslant 0} t^{d i} S^{i}\left(q_{d}\right)\right)\right]\left[\prod_{d \text { odd }}\left(\sum_{i \geqslant 0} t^{k i} \lambda^{i}\left(q_{d}\right)\right)\right], \\
& \widehat{w}\left(q_{1}, q_{2}, \ldots, q_{n}, \ldots\right)=\left\{\sum_{d \mid n}(-1)^{n(m / n-1)} d \Psi^{n / d}\left(q_{d}\right)\right\}_{n \in \mathbb{N}} .
\end{aligned}
$$

COROLLARY 8. $\widehat{\operatorname{Lie}}_{n}=s g n_{\mathbb{S}_{n}}\left(\operatorname{Lie}_{n}\right)$, for $n \geqslant 1$.

Proof: It is easy to see that $\widehat{w}^{-1}: \operatorname{Im} \widehat{w} \rightarrow \widehat{R}^{\mathbf{N}}$ is defined by:

$$
\widehat{w}^{-1}\left(a_{1}, a_{2}, \ldots, a_{n}, \ldots\right)=\left\{1 / n \sum_{d \mid n}(-1)^{(n-1) n / d} \mu(d) \Psi^{d}\left(a_{n / d}\right)\right\}_{n \in \mathbb{N}}
$$

Now we have

$$
\mathrm{T}=\sum_{b \geqslant 0} s_{1}^{n} \text { and } \widetilde{e}^{-1}\left(\sum_{n \geqslant 0} s_{1}^{n} t^{n}\right)=\left\{1 / n \sum_{d l n}(-1)^{(n-1) n / d} \mu(d) s_{d}^{n / d}\right\}_{n \in \mathbb{N}},
$$

which implies:

$$
\widehat{\operatorname{Lie}}_{n}=1 / n \sum_{d \mid n}(-1)^{(n-1) n / d} \mu(d) s_{d}^{n / d}, \text { for } n \geqslant 1
$$

To get the desired formula it suffices to point out that $\operatorname{sgn}_{\mathbb{S}_{n}}\left(s_{d}^{n / d}\right)=(-1)^{(n-1) n / d}$, for $d, n \in \mathbb{N}$ such that $d \mid n$.

\section{REFERENCES}

[1] A. Dress and C. Siebeneicher, 'The Burnside ring of the infinite cyclic group and its relations to the necklace algebra, $\lambda$-ring and the Universal ring of Witt vectors', $A d v$. in Math. 78 (1989), 1-41. 
[2] M. Gerstenhaber and S. Schack, 'A Hodge type decomposition for commutative algebra homology', J. Pure Appl. Algebra 48 (1987), 229-247.

[3] P. Hanlon, "The action of $S_{n}$ on the components of the Hodge decomposition of Hochschild homology', Michigan Math. J. 37 (1990), 105-124.

[4] A. Klyachko, 'Lie elements in the tensor algebra', Siberian Math. J. 15 (1974), 1296-1304.

[5] D. Knutson, ' $\lambda$-ring and the representation theory of the symmetric groups IV', Lecture Notes in Mathematics 308 (Springer-Verlag, Berlin, Heidelberg, New York).

[6] J.-L. Loday, 'Opérations sur l'homologie cyclique des algèbres commutatives', Invent. Math. 96 (1989), 205-230.

[7] N. Metropolis and G-C. Rota, 'The Cyclotomic identity', Contemp. Math. 34 (1984), 19-24.

[8] J. Kerber, 'The representation theory of the symmetric group', Encyclopedia of Mathematics (Cambridge University Press, Cambridge New York).

Depto. de Matemáticas

Facultad de Ciencias Exactas y Nat.

Universidat de Buenos Aires

Argentina 